\title{
Rare neurological presentations of Dengue Fever
}

\section{Navgeet Mathur $M D^{1}$, Medha Mathur $M D^{2}$}

${ }^{1}$ Department of General Medicine, Geetanjali Medical College and Hospital, Udaipur, Rajasthan, India, ${ }^{2}$ Department of Community Medicine, Geetanjali Medical College and Hospital, Udaipur, Rajasthan, India.

\section{Abstract}

Dengue fever may present with variety of neurological manifestations. We report two cases of such unusual neurological presentations. One case is of dengue fever induced bilateral abducens nerve palsy and the other of dengue cerebellitis. In both these cases as reported in previous studies, imaging studies have been normal and nonspecific. These presentations may possibly have immunological etio-pathogenesis and steroids may be helpful.

Key words: abducens nerve, cerebellitis, dengue, palsy

\section{Introduction}

$\mathrm{D}$ eveloping countries like India still face high morbidity and mortality due to mosquito borne diseases. Dengue is one of the mosquito-borne disease having dreadful complications. Dengue has variety of presentation although it mainly presents with fever, joints pain, icterus and hemorrhagic manifestations due to thrombocytopenia. Neurological manifestations of dengue are less common. Neurological manifestations of dengue include encephalopathy, encephalitis, aseptic meningitis, stroke, parkinsonism, neuromuscular complications and immune-mediated neurological syndromes like mononeuropathies, polyradiculoneuritis, Guillain Barre Syndrome (GBS), brachial neuritis, transverse myelitis,

Access this article online
Website: https://www.nepjol.info/index.php/NJN
DOI: https://doi.org/10.3126/njn.v18i1.34101
HOW TO CITE
Mathur N, Mathur M. Rare neurological presentations of Dengue
Fever. NJNS. 2021;18(1):66-69.

${ }^{1}$ ORCID id: 0000-0003-0610-361X

${ }^{2}$ ORCID id: 0000-0001-9539-5890

\author{
Address for correspondence: \\ Dr. Navgeet Mathur, \\ Department of General Medicine, \\ Geetanjali Medical College and Hospital, \\ Udaipur, Rajasthan, India-313001 \\ E-mail: mathurdrnavgeet@gmail.com \\ Phone: +91-9610653520
}

Copyright (C) 2021 Nepalese Society of Neurosurgeons (NESON)

ISSN: 1813-1948 (Print), 1813-1956 (Online)

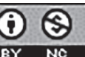

This work is licensed under a Creative Commons

Attribution-Non Commercial 4.0 International License. acute disseminated encephalomyelitis (ADEM), acute cerebellitis, opsoclonus, myoclonus. ${ }^{1}$ We present two rare cases of dengue with different neurological presentations, and discuss on diagnosis and treatment approach.

\section{Case Report}

First case, an 18 years old Hindu unmarried female who reported to health facility in August 2017 and was a resident of Udaipur, Rajasthan. She was involved in household responsibilities. She had fever for three days. Fever was mild to moderate, intermittent in nature associated with body ache. After two days of onset of fever, she developed insidious onset blurring of vision on bilateral lateral gaze, however there was no blurring of vision on central, upward, downward gaze.

There was no history of cough, burning micturition, vomiting, diarrhea, abdominal pain, chronic headache, chills and rigor, redness of eyes, excessive lacrimation, evening rise of temperature, excessive sweating, loss of consciousness, altered behavior, limb weakness, chest pain, palpitation, blood loss, trauma, seizure episode, involuntary bladder and bowel evacuation, constipation, urinary retention, ear discharge, hearing impairment, skin lesion, recent weight gain or loss, difficulty in breathing or swallowing, hoarseness of voice, poisoning, insect or snake bite.

There was no history of diabetes, hypertension, tuberculosis, psychiatric illness, trauma, operative procedure or similar illness in past except for menometrorrhagia. She was vegetarian, non-smoker and non-alcoholic.

Systemic examination was non-significant, except for bilateral weakness of lateral movement of eyes suggestive of bilateral sixth cranial nerve paresis. Other cranial nerves examination was normal. Signs of meningitis and 


\section{Rare neurological presentations of Dengue Fever}

cerebellar lesion were absent. Gait was normal.

Laboratory investigation revealed anaemia $(\mathrm{Hb}$ with $9.8 \mathrm{mg} / \mathrm{dl}$ ), normal counts (TLC $8,600 / \mathrm{mm}^{3}$ ) and low platelets $\left(145,000 / \mathrm{mm}^{3}\right)$. Anemia was normocytic normochromic and ESR was $30 \mathrm{~mm}$. Blood sugar, renal and liver function test, serum electrolytes were within normal limits. No significant abnormality was found in ECG, chest X-ray, ultrasonography of abdomen. Malaria antigen detection test by solid phase immunochromatography was negative. No species of malaria parasite was visible on peripheral blood film examination. Dengue IgM antibodies was found positive, done by lateral flow immunochromatographic assay. VDRL test, Widal titer, Typhoid IgM/IgG antibodies, $\mathrm{HIV}$ immunochromatographic assay, $\mathrm{HBsAg}$, anti $\mathrm{HCV}$ antibodies, ANA, T3, T4, TSH were within normal limits. No abnormality was found in CSF examination and contrast enhanced MRI brain study.

After one day of hospital admission she had additional complaint of diplopia with conversion of eyes (Figure 1). Systemic examination was repeated. She had conversion of eyes with complete restriction of lateral movement of both eyes suggestive of bilateral lateral rectus muscle palsy. On repeated fundus examination, no significant abnormality was found. Platelet count improved to $173,000 / \mathrm{mm}^{3}$ on the next day of hospital admission.

Patient was kept on symptomatic treatment along with injectable ceftriaxone to treat and prevent secondary bacterial infections. Steroid was given as injectable dexamethasone $4 \mathrm{mg}$ thrice a day to control and prevent further immunological injury to the brain. During the course of admission patient improved. After three days of admission fever subsided and there was significant improvement in lateral movement of eyes. Steroid was tapered down. After six days of admission, patient completely recovered of fever and had normal eye movements (Figures 2, 3, 4). Patient was discharged on medication supplementing Iron, folic acid and vitamin B complex only. On regular follow-up of more than 6 months no abnormal neurological sign and symptoms reappeared.

Second case was a 41 years old married Hindu male who reported to us in August, 2013. He was resident of Jaipur, Rajasthan and used to work in a grocery store. He presented with history of fever with body ache for 6 days and difficulty in standing, walking with imbalance and swaying of body for last 3 days. Fever was mild to moderate, non-documented, intermittent in nature associated with body ache. After three days of onset of fever, he developed insidious onset gradually progressive swaying of body in upright posture with difficulty in standing and walking. Initially patient had low amplitude swaying of body although he was able to stand and walk with some difficulty. Over next 48 hours he was unable to walk and able to stand only by difficulty with large amplitude swaying of body. He needed assistance and support for using wash room and other ambulatory activities. There was no difficulty in holding objects, buttoning -unbuttoning of shirt and combing of hair. There was no history of slippage of footwear but he had difficulty in climbing upstairs and standing up from squatting position.

There was no history of cough, burning micturition, chills and rigor, evening rise of temperature, chest pain, palpitation, excessive sweating, loss of consciousness, altered behavior, vomiting, diarrhea, abdominal pain, blood loss, trauma, seizure episode, involuntary bladder bowel evacuation, constipation, urinary retention, vision loss, hearing impairment, skin lesions, recent weight gain or weight loss, difficulty in breathing or swallowing, hoarseness of voice, loss of sensations, poisoning or binge alcohol intake.

There was no relevant past history. He was a vegetarian and had history of occasional smoking ( 1 cigarette pack in a month). Vitals were within normal limits. On systemic examination of nervous system higher mental functions were normal with intact cranial nerve examination except upbeat nystagmus. On motor examination bilateral bulk, tone, power of muscles was normal. Bilaterally deep and superficial reflexes were normal. No abnormality was found on sensory examination. Signs of meningitis were absent. On examination of cerebellar signs, upbeat nystagmus was present with swaying of body with no predominant direction. Romberg's sign and tremors were absent. However, coordination was impaired. No significant abnormality was found on examination of other systems.

On routine investigation hemoglobin, total leucocytes count and platelet counts were within normal limits. His peripheral blood film, ESR, blood sugar, renal-liver function test and serum electrolytes were within normal limits. No significant abnormality was found in ECG, chest X-ray and ultrasonography of abdomen. Malaria antigen detection test by solid phase immunochromatography was negative. No species of malaria parasite was visible on peripheral blood film examination. Dengue NS1 antigen was found positive performed by lateral flow immunochromatographic assay. VDRL test, Widal titre, typhoid IgM/IgG antibodies, $\mathrm{HIV}$ immunochromatographic assay, $\mathrm{HBsAg}$, anti $\mathrm{HCV}$ antibodies, anti-nuclear antibodies (ANA) were found within normal values. CSF examination and contrast enhanced MRI brain were normal.

Subsequently he was managed as a case of dengue cerebellitis. He was treated with symptomatic treatment, 


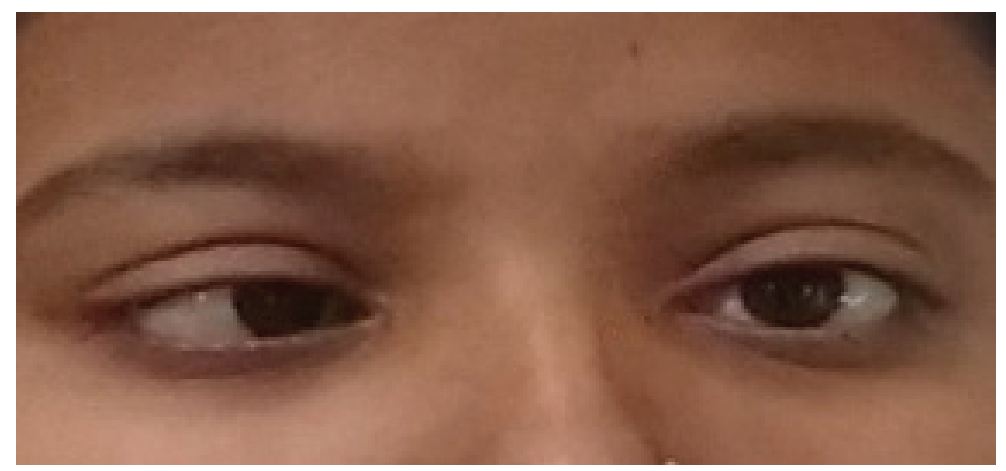

Figures 1: Convergence of eyes with bilateral sixth cranial nerve palsy
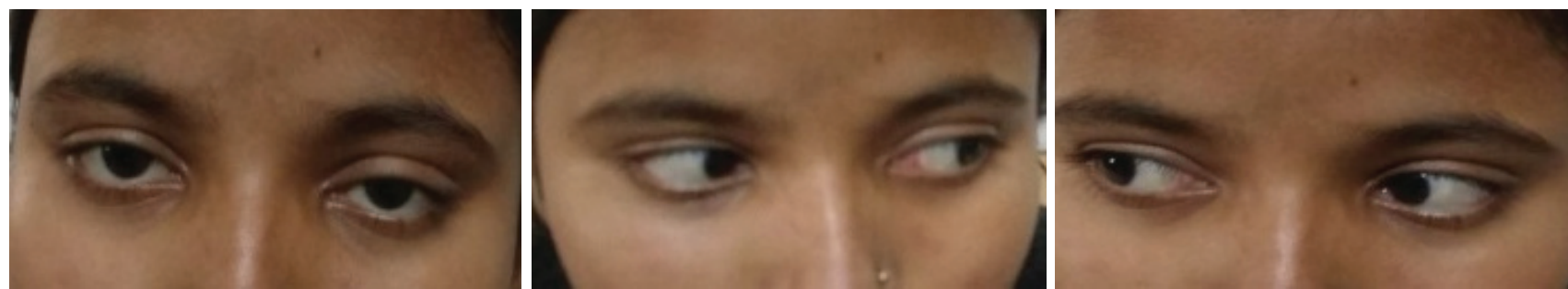

Figures 2, 3, 4: Follow-up showed completely improved convergence and eye movements (after treatment)

intravenous fluids, injectable ceftriaxone to treat and prevent secondary bacterial infections. Steroid was given in form of injectable dexamethasone $4 \mathrm{mg}$ thrice a day to control and prevent further immunological injury to the brain. During the course of admission patient improved. After three days of admission fever subsided and there was significant improvement in swaying of body. Patient was able to stand, walk and use washroom without any support. Only very low amplitude swaying of body was there. Patient was discharged on oral prednisolone 40 mg once daily for five days. On follow up after five days patient completely recovered. Steroid was tapered down and stopped later. On regular follow up of more than a year no abnormal neurological sign and symptoms reappeared.

\section{Discussion}

These two cases highlighted the rare presentations of dengue. Various neurological manifestations in background of fever especially in seasons favorable for mosquito borne diseases, warrants the need of keeping dengue as one of the etiological possibilities.

Dengue induced abducens nerve palsy has been mentioned in very few case reports ${ }^{2-4}$ and all these case reports had unilateral abducens nerve palsy. After extensive search, no case was found reporting bilateral involvement. At the best of our knowledge current case study is the first ever case reported worldwide of dengue induced bilateral abducens nerve palsy.
Only few cases of dengue cerebellitis were reported previously. ${ }^{5-9}$ Some of them also had normal MRI with definite signs and symptoms of cerebellitis ${ }^{5,6}$ like current study while some ${ }^{7,8}$ found abnormalities on imaging. This underlines the fact that MRI brain may be normal in case of dengue cerebellitis.

Previous studies regarding dengue induced abducens nerve palsy and cerebellitis also mentioned complete recovery of sign and symptoms like current study. Use of steroid as treatment was not clearly mentioned in previous studies. In this case series steroid was used due to a possibility of immunological etio-pathogenesis. Bilateral involvement and no focal lesion on imaging also favor this fact. In current study recovery of patients was faster than mentioned in previous studies especially in case of dengue induced abducens nerve palsy. This may possibly be due to early use of steroids.

\section{Conflict of Interest: None Source(s) of support: None}

\section{References}

1. Carod-Artal FJ, Wichmann O, Farrar J, Gascón J. Neurological complications of dengue virus infection. Lancet Neurol. 2013;12(9):906-919. https://doi. org/10.1016/S1474-4422(13)70150-9

2. Shivanthan M.C., Ratnayake E.C., Wijesiriwardena B.C. et al. Paralytic squint due to abducens nerve 


\section{Rare neurological presentations of Dengue Fever}

palsy: a rare consequence of dengue fever. BMC Infect Dis. 2012;12:156. https://doi.org/10.1186/14712334-12-156

3. Mazliha M, Boo YL, Chin PW. Isolated unilateral sixth cranial nerve palsy: A rare presentation of dengue fever. Malays Fam Physician. 2016;11(1);2526. https://pubmed.ncbi.nlm.nih.gov/28461846/

4. Mishra A, Shukla S, Aggarwal S, et al. Lateral rectus palsy in a case of dengue fever. Med J Armed Forces India. 2013;1:10-2. https://doi.org/10.1016/j. mjafi.2013.05.010

5. Withana, M., Rodrigo, C., Chang, T. et al. Dengue fever presenting with acute cerebellitis: a case report. .2014;7:125. https://doi.org/10.1186/17560500-7-125

6. Singh R, Pandey P, Manchanda BV, Chauhan R, Vasishta S, Sharma S. A Case Report of Acute Cerebellitis as a presenting feature of Dengue Fever.
IOSR Journal of Dental and Medical Sciences. 2017;16(8):43-44. https://doi.org/10.9790/08531608054344

7. Patel ML, Shyam R, Pushkar DK, Sachan R. Dengue encephalitis with cerebellar involvement: A rare case report. Ann Trop Med Public Health. 2017;10:734-6. https://www.atmph.org/text. asp?2017/10/3/734/213181

8. Karunarathne S, Udayakumara Y, Fernando $\mathrm{H}$. Epstein-Barr virus coinfection in a patient with dengue fever presenting with post-infectious cerebellitis: a case report. J Med Case Rep. 2012;6:43. https://doi. org/10.1186/1752-1947-6-43

9. Weeratunga PN, Caldera HP, Gooneratne IK, Gamage R, Perera WS, Ranasinghe GV, et al. Spontaneously resolving cerebellar syndrome as a sequelae of dengue viral infection: A case series from Sri Lanka. Pract Neurol. 2014;14:176-8. https://doi.org/10.1016/j. tmaid.2013.11.001 